\title{
Pulmonary Embolism due to Right Ventricular Thrombus in a Case of Behcet's Disease
}

\author{
Masanori Yasuo, MD; Satoshi Nagano, MD; Yoshikazu Yazaki, MD; Tomonobu Koizumi, MD; \\ Hiroshi Kitabayashi, MD; Hiroshi Imamura, MD; Jun Amano, MD*; Mitsuaki Isobe, MD
}

\begin{abstract}
Right ventricular thrombus is a very rare manifestation of cardiovascular Behcet's disease. A 25-year-old man was admitted to hospital due to cough and fever of unknown origin. He experienced repetitive pulmonary embolism due to a right ventricular thrombus, which was surgically removed. A diagnosis of Behcet's disease was made based on his clinical course and the histological findings of the right ventricular wall and the skin lesion. He was quickly relieved of his symptoms after warfarinization and cyclosporine therapy. (Jpn Circ J
\end{abstract} 1999; 63: 909-911)

Key Words: Behcet's disease; Pulmonary embolism; Right ventricular thrombus

B ehcet's disease is now recognized to be a systemic disorder that mainly affects young adults in Mediterranean, Middle Eastern and Far Eastern countries. Various cardiovascular manifestations have been reported, but all are rare. We describe a patient with this disease who presented with the unusual clinical manifestation of a right ventricular thrombus associated with multiple bilateral pulmonary embolism.

\section{Case Report}

A 25-year-old man was admitted due to cough, fever of unknown origin and generalized arthralgia that had lasted for 3 months. He had a dry cough during the hospitalization. A chest X-ray on admission showed bilateral infiltration and consolidation (Fig 1). The clinical signs and symptoms were not improved by antibiotics and responded only to steroids, but the high fever and arthralgia recurred during withdrawal from the latter. He had a history of thrombophlebitis of his right leg 1 year before admission and recurrent oral ulcers for several years. He was transferred for further investigation.

On admission the erythrocyte sedimentation rate was 87 $\mathrm{mm} / \mathrm{h}$, the white blood cell count was $13,710 / \mathrm{ml}$ and Creactive protein was $19.83 \mathrm{mg} / \mathrm{dl}$. IL-6 was elevated to 31.6 $\mathrm{pg} / \mathrm{ml}$. HLA B51 antigen was not present. Anticardiolipin antibodies, lupus anticoagulant, C- and P-antineutrophil cytoplasmic antibody were absent. His laboratory data are shown in Table 1. The electrocardiogram showed right axis deviation, and echocardiography demonstrated a $20 \times 20$ $\mathrm{mm}$ right ventricular apical solid mass (Fig 2). This mass lesion had a wide peduncle and grape-like clusters, but did not show any movement. The systolic and diastolic function of both ventricles were determined to be normal. No cardiac enlargement was found. A thoracic computed

(Received April 14, 1999; revised manuscript received July 23, 1999; accepted July 26, 1999)

First Department of Internal Medicine and *Second Department of Surgery, Shinshu University School of Medicine, Matsumoto, Japan Mailing address: Mitsuaki Isobe, MD, First Department of Internal Medicine, Shinshu University School of Medicine, 3-1-1 Asahi, Matsumoto 390-8621, Japan. E-mail: isobemi@hsp.md.shinshu-u. ac.jp tomography (CT) scan showed multiple, ill-defined, faint infiltration shadows in the bilateral inferior and middle lung lobes (Fig 3B) and a contrast CT scan also showed a mural mass in the right ventricle (Fig 3A). Pulmonary perfusion scintigraphy demonstrated multiple bilateral defects (Fig 4A, B), but the ventilation scan was normal (Fig 4C). Examination of bronchoalveolar lavage fluid was not suggestive of either infection or bronchiolitis obliterans with organizing pneumonia.

The abnormal shadow in the lung field, the cough and the high fever were considered to be caused by pulmonary embolism due to a right ventricular mass that enlarged during the 2 weeks of observation. We could not rule out the possibility that the intracavity mass could be septic emboli or a neoplasm such as cardiac myxoma. Therefore, surgery to remove the mass was undertaken to prevent recurrence of the pulmonary embolization.

The mass was an intramural thrombus with a rough surface and it was firmly adhered to the right ventricular wall. Massive fibrin and cellular infiltration was observed

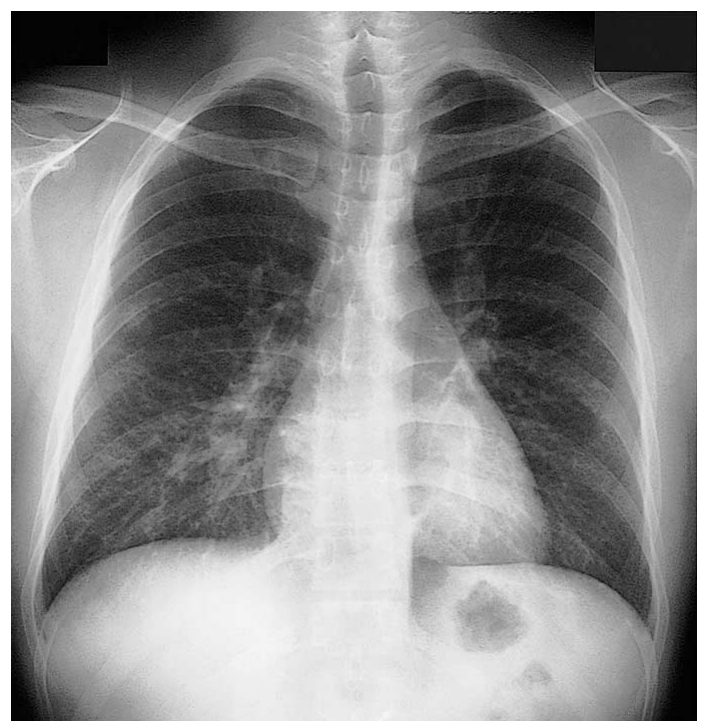

Fig 1. Chest X-ray on admission showing bilateral infiltration and consolidation. 
Table 1 Laboratory Data

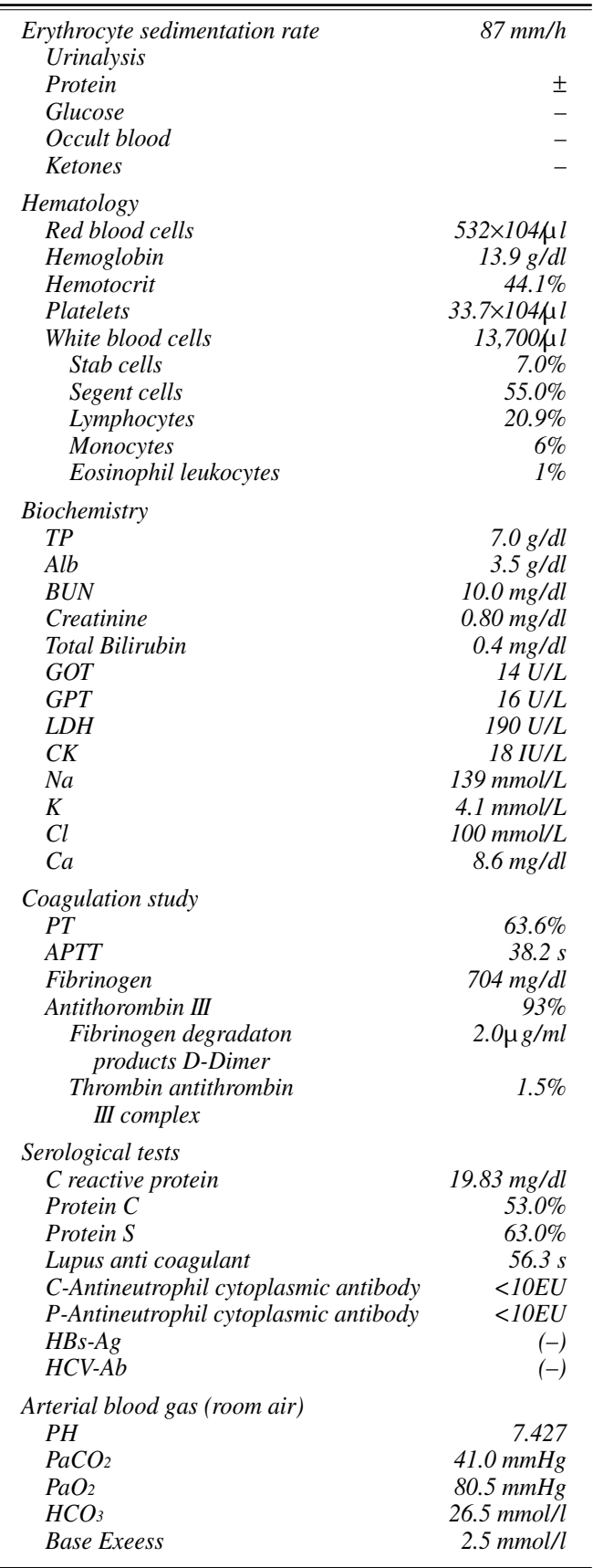

in the thrombus on histology (Fig 5A). Endocarditis of the adjacent right ventricular wall with massive neutrophil infiltration was also noted (Fig 5B). Pulmonary tissue obtained from the infiltrative lesion was found to be fibrotic, indicating the existence of pulmonary infarction. The post-surgical course was uneventful except for sustained high fever. After warfarinization was started, his pulmonary symptoms disappeared. During the hospitalization thrombophlebitis recurred on his left dorsal pedicle and this lesion was histologically confirmed to be erythema nodosa delineated by intravenous thrombus, circumvascular inflammation and steatitis. A clinical diagnosis of Behcet's disease was made. After starting cyclosporine (starting dose of $300 \mathrm{mg} / \mathrm{day}$ ) he was quickly relieved of his symptoms. The C-reactive protein reduced to within

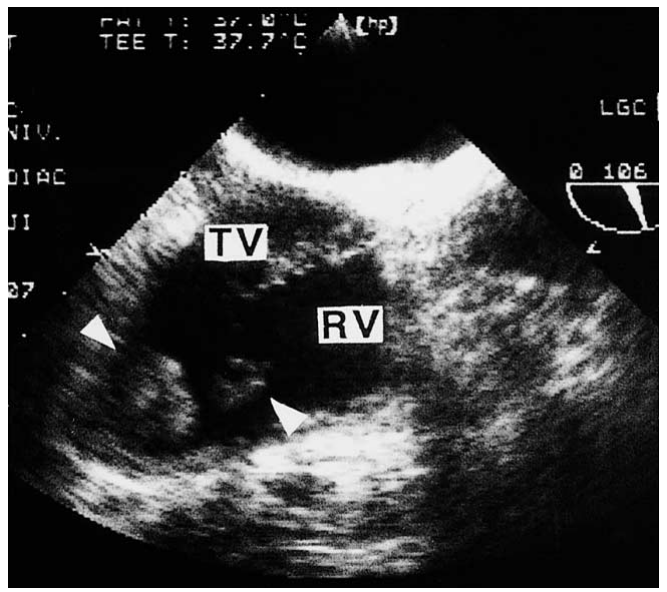

Fig 2. Echocardiography showing a $20 \times 20 \mathrm{~mm}$ mass and a smaller mass in the right intraventricular apex. This mass lesion had a wide peduncle and grape-like clusters. RV, right ventricle, TV, tricuspid valve.

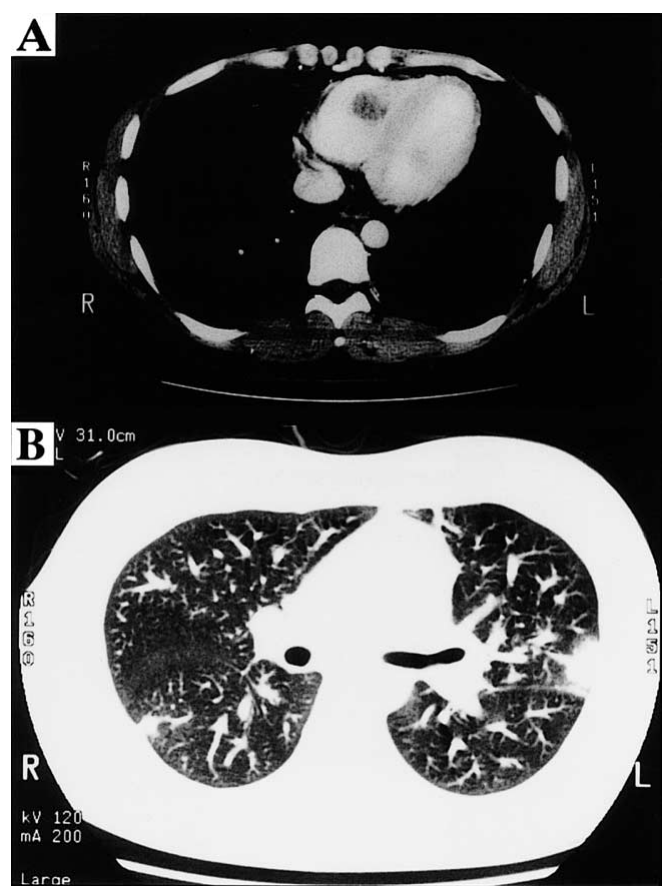

Fig 3. Thoracic computed tomography showing (A) an intracavity mass in the right ventricle and (B) infiltration shadows in the bilateral inferior lung lobes.

normal range. Thereafter his clinical course was excellent. However, 1 year later he suffered from left hemiparesis, which was caused by neuro-Behcet's disease diagnosed by magnetic resonance imaging.

\section{Discussion}

Cardiac manifestations of Behcet's disease are rare, and include aortic regurgitation due to aortitis, pericarditis, myocarditis, conduction-system abnormality, and coronary artery occlusion, ${ }^{1}$ though Ozkan et al mentioned that 65 patients with this disease did not show an increased prevalence of echocardiographic abnormalities compared with their controls. Although intracardiac thrombi are known to be complications of vasculo-Behcet's disease, right ventric- 

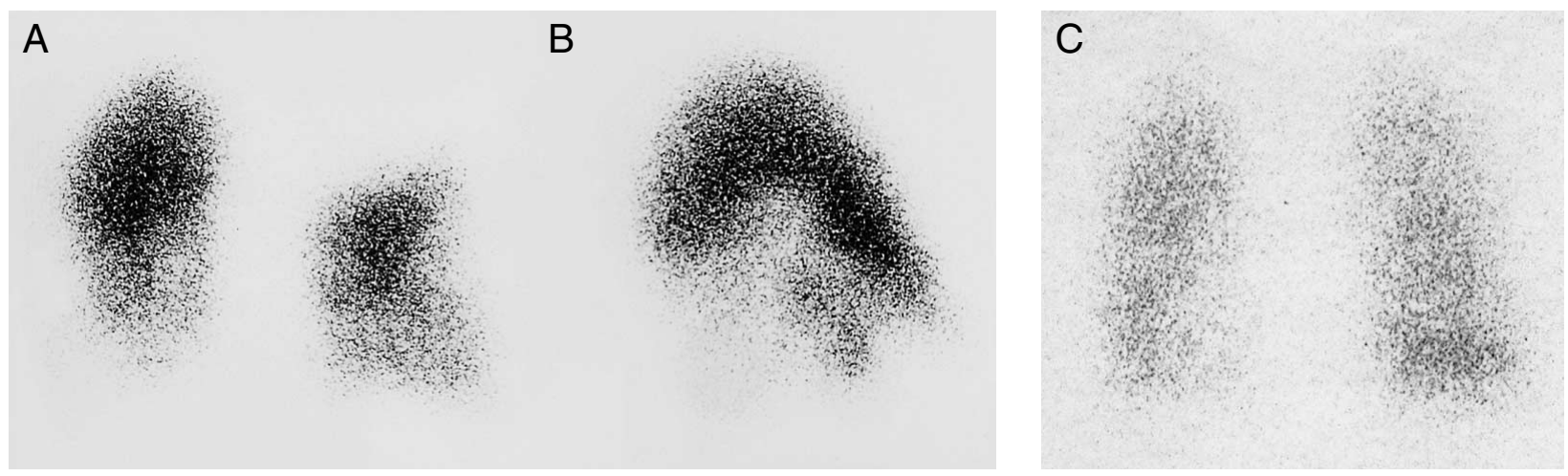

Fig 4. Pulmonary perfusion scintigraphy showing multiple bilateral defects at right upper lobe and left inferolateral lung field (A, B), with a normal ventilation scan $(\mathrm{C})$, suggesting the existence of pulmonary artery obstruction.
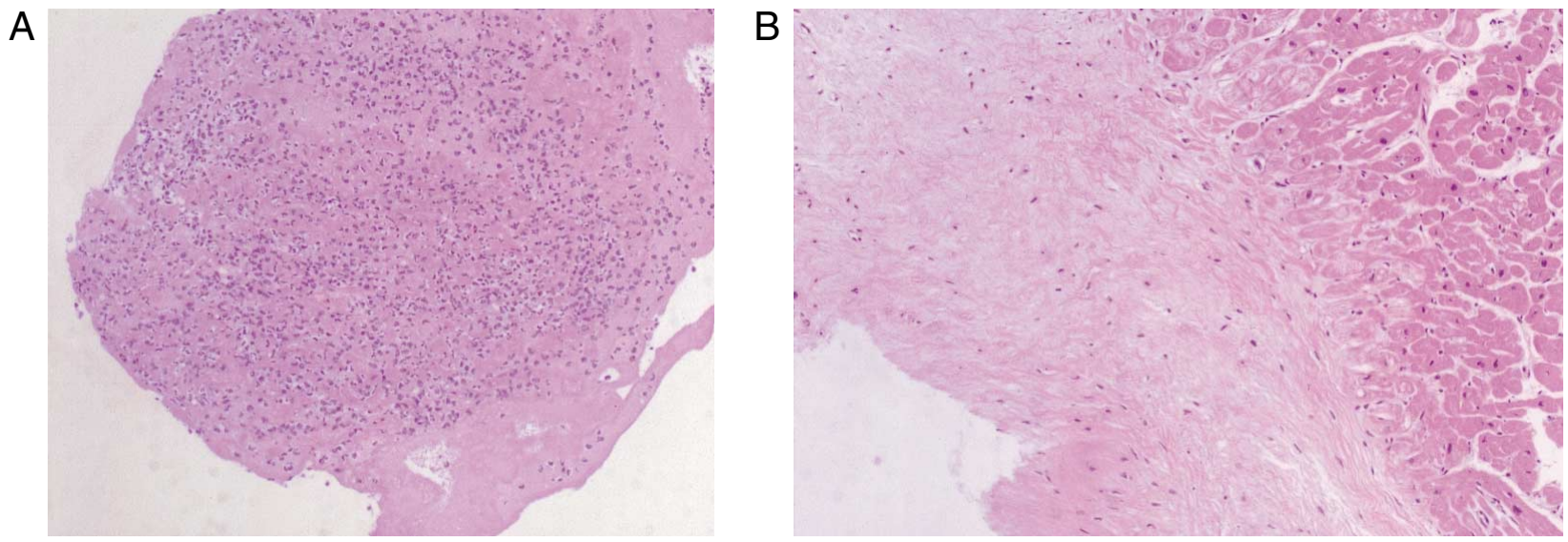

Fig 5. Photomicrographs of the right ventricular mass hematoxylin-eosin (H\&E). (A) Massive fibrin and cellular infiltration were observed in the thrombus. (B) Endocarditis of the adjacent right ventricular wall. Neutrophil infiltration was also noted $(\mathrm{H} \& \mathrm{E}, \times 50)$.

ular thrombi are extremely rare. Only 10 cases have been reported to date in the English literature ${ }^{3-11}$ As well, pulmonary involvement with this disease is rare, with the usual clinical presentation of pulmonary involvement being recurrent massive hemoptysis 10,12 Repetitive pulmonary embolism has been reported in only 3 cases?.,12

In the present case, the differential diagnoses for the pulmonary thromboembolism were myocarditis, thrombophlebitis, infective endocarditis and carcinoma of the kidney or liver. Myocarditis was ruled out based on the histological findings of the cardiac tissue, the electrocardiogram and the echocardiographically determined cardiac performance. The abdominal CT scan and gallium scintigraphy did not show any internal malignancy. The absence of active pulmonary arteritis was also confirmed by histology. Because the patient's pulmonary symptoms disappeared after the surgical resection of the intracavity mass, which was before the disappearance of his thrombophlebitis, it is unlikely that the thrombophlebitis of his leg caused the pulmonary embolism. His clinical course and repeated blood culture were not suggestive of infective endocarditis. On the basis all these findings, his pulmonary involvement is considered to be caused by repeated embolisms resulted from right ventricular mural thrombi. It is reasonable to assume that the local endocarditis associated with Behcet's disease increases the thrombogenicity of the endocardial wall. We conclude that Behcet's disease should be considered within the spectrum of diseases that cause pulmonary embolism and intracavity masses of the right heart.

\section{References}

1. Coblyn JS, Weinblatta ME: Rheumatic diseases and the heart. In: Braunwald E, editor. Heart disease, 5th edn. Philadelphia: WB Saunders, 1997: 1782-1783

2. Ozkan M, Emel O, Ozdemir M, Yurdakul S, Kocak H, Ozdogan H, et al: M-mode, 2-D and Doppler echocardiographic study in 65 patients with Behcet's syndrome. Eur Heart J 1992; 13: 638-641

3. Erol ICC, Sonel A, Akalin H: Behcet's disease: cardiac and pulmonary involvement. Eur Heart J 1986; 7: 999-1002

4. Augarten A, Apter S, Theodor R: Right ventricular thrombus and pulmonary arteritis in Behcet's disease. Isr J Med Sci 1987; 23: 900 901

5. Sayin AG, Vural FS, Bozkurt AK, Oz B, Uygun N: Right atrial thrombus mimicking myxoma and bilateral pulmonary arteritis in Behcet's disease. Angiology 1993; 44: 915-918

6. Madanat WY, Prokaeva TB, Kotelínikova GP, Alekberova ZS Endocarditis with left atrial thrombus formation in Behcet's disease mimicking myxoma. J Rheumatol 1993; 20: 1982-1984

7. Huong DLT, Dolmazon C, Zuttere DD, Wechsler B, Godeau P, Piette J-C: Complete recovery of right intraventricular thrombus and pulmonary arteritis in Behcet's disease. Br J Rheumatol 1997; 36: $130-132$

8. Koc Y, Gullu I, Akpek G, Akpolat T, Kiraz S, Batman F, et al: Vascular involvement in Behcet's disease. J Rheumatol 1992; 19: $402-410$

9. Vanhaleweyk G, El-Ramahi KM, Hazmi M, Sieck JO, Zaman L, Fawzy M: Right atrial, right ventricular and left ventricular thrombi in (incomplete) Behcet's disease. Eur Heart J 1990; 11: 957-959

10. Lie JT: Cardiac and pulmonary manifestations of Behcet syndrome. Pathology, Research and Practice 1988; 183: 347-352.

11. El-Ramahi KM, Fawzy ME, Sieck JO, Vanhaleweyk G: Cardiac and pulmonary involvement in Behcet's disease. Scand J Rheumatol 1991; 20: $373-376$

12. Davies JD: Behcet syndrome with haemoptysis and pulmonary lesions. J Pathol 1973; 109: 351-356 